\title{
La importancia de la determinación del HER2 en el cáncer gástrico avanzado: a propósito de un caso clínico
}

\section{Importance of HER2 status determination in advanced gastric cancer: A case study}

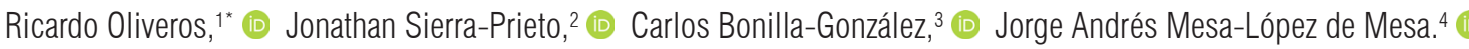

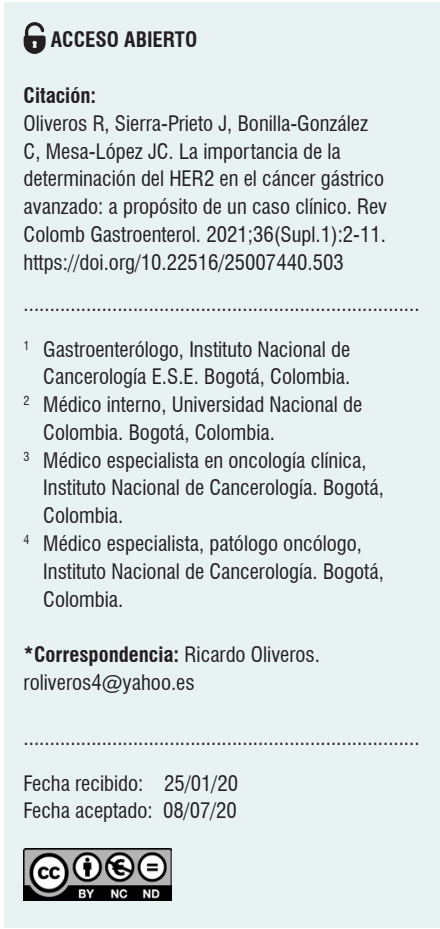

\begin{abstract}
Resumen
El cáncer gástrico avanzado es una entidad que incluye dos situaciones clínicas distintas: el cáncer gástrico localmente avanzado no resecable y la enfermedad metastásica, cuyo tratamiento estándar es la quimioterapia. La sobreexpresión del receptor 2 del factor de crecimiento epidérmico humano (HER2) se puede presentar en esta enfermedad de un $9 \%$ a un $38 \%$ y ha sido el primer biomarcador predictivo utilizado para el tratamiento dirigido con trastuzumab en pacientes con tumores gástricos y de la región gastroesofágica avanzados. Se presenta en este artículo el caso de un paciente con cáncer gástrico avanzado con HER2 positivo manejado con quimioterapia convencional más trastuzumab como terapia blanco con adecuada respuesta clínica.
\end{abstract}

\section{Palabras clave}

Cáncer gástrico avanzado, manejo, trastuzumab, HER2.

\section{Abstract}

Advanced gastric cancer (AGC) is an entity that encompasses two distinct clinical situations: locally advanced unresectable gastric cancer and metastatic disease, with chemotherapy as the standard treatment. HER2 overexpression can occur in $9 \%$ to $38 \%$ of the cases with this disease and has been the first predictive biomarker used for trastuzumab-targeted therapy in patients with advanced gastric and gastroesophageal tumors. This article presents a patient with AGC and positive HER2 treated with conventional chemotherapy plus trastuzumab as targeted therapy with adequate clinical response.

\section{Keywords}

Advanced gastric cancer; Treatment; Trastuzumab; HER2.

\section{INTRODUCCIÓN}

El cáncer gástrico es un problema de salud pública. Cada año en el mundo se presentan más de 1 millón de casos nuevos y cerca de 850000 fallecidos. En Colombia, según Globocan 2018, se estiman 7419 casos nuevos al año, que representan el tercer lugar en frecuencia $(7,3 \%)$ y la primera causa de mortalidad por cáncer con 5505 casos (13,7\%) (1).
En los países occidentales, aproximadamente el $50 \%$ de los pacientes con cáncer gástrico que consultan por primera vez presenta enfermedad metastásica y en estos estadios la supervivencia global a 5 años es menor al $5 \%$ (2).

El cáncer gástrico tiene varias clasificaciones. Una de ellas menciona al cáncer gástrico como una enfermedad localizada (estadios 0, I, II y III resecable) y como una enfermedad avanzada y metastásica (estadio IV irresecable). Esta 
última (cáncer gástrico avanzado) incluye dos situaciones: el cáncer gástrico localmente avanzado no resecable y la enfermedad metastásica (3).

El tratamiento de primera línea para el cáncer gástrico avanzado es la quimioterapia con la combinación de las fluoropirimidinas y el platino tales como el 5-fluorouracilo (5FU) más cisplatino (FP) o la capecitabina más cisplatino (XP) (4). Estos esquemas han demostrado mejorar la supervivencia global, la supervivencia libre de progresión y la calidad de vida cuando se compara con el mejor cuidado de soporte (3).

El receptor 2 del factor de crecimiento epidérmico humano (HER2) es un miembro de la familia EGRF (receptores del factor de crecimiento epidérmico) que está asociado con incremento en la división, proliferación, diferenciación y apoptosis de las células tumorales de los cánceres de la glándula mamaria, estómago y otros tumores sólidos (5).

Varios estudios han demostrado que los carcinomas de mama HER2 positivo son más agresivos comparados con aquellos HER2 negativos y están asociados con un incremento en el riesgo de crecimiento local y de metástasis a distancia (6). El HER2 también está sobreexpresado en carcinomas gástricos y de la unión gastroesofágica $(6,7)$. La sobreexpresión del HER2 en el cáncer gástrico podría resultar en tumores que tienen mayor capacidad proliferativa e invasora (6).

Se ha evaluado la relación entre el estado HER2 y el pronóstico de los pacientes con cáncer gástrico. Algunos han demostrado que el HER2 positivo estuvo asociado con un significativo peor pronóstico, mientras que otros no han encontrado ninguna asociación; sin embargo, la supervivencia global fue mayor en los pacientes con HER2 positivo comparado con los pacientes con tumores HER2 negativo. Por esto, la relación entre el estado HER2 y el pronóstico permanece en controversia (6).

El HER2 es un importante biomarcador predictivo en los tumores gástricos y de la unión gastroesofágica, y el análisis del estado del HER2 por técnicas de inmunohistoquímica (IHC) y de hibridación con fluorescencia in situ (FISH) utilizando diferentes métodos de puntaje sugieren que el HER2 está sobreexpresado en el $7 \%-34 \%$ de los tumores gástricos. Los resultados favorables derivados del estudio TOGA se correlacionan con el nivel de sobreexpresión del HER2 $(8,9)$.

Con el descubrimiento de la sobreexpresión del HER2 en pacientes con cáncer gástrico avanzado, se ha recomendado adicionar a la quimioterapia el trastuzumab, un medicamento anti-HER2 en la primera línea de tratamiento que ha demostrado mejorar los desenlaces de eficacia $(7,10)$. Actualmente, la exactitud en el resultado y estandarización de la prueba HER2 es crucial para identificar la población blanco para el tratamiento y beneficio con este medicamento (11).

La introducción del trastuzumab ha permitido el establecimiento de una nueva enfermedad el cáncer gástrico HER2 positivo, similar al caso del cáncer de mama HER2 positivo (6). El acceso al estado del HER2 en los pacientes con cáncer gástrico es ahora vital para ayudar a la toma de decisiones para asegurar que el paciente reciba el mejor tratamiento posible (6). Por este motivo es importante que todos los pacientes con cáncer gástrico avanzado o de la unión gastroesofágica deban ser examinados para determinar el estado de HER2 (6).

El objetivo de este artículo es presentar el caso de un paciente con cáncer gástrico avanzado metastásico con HER2 positivo, manejado con quimioterapia más trastuzumab con respuesta clínica y supervivencia favorables.

\section{PRESENTACIÓN DEL CASO}

Se presenta el caso clínico de un paciente masculino de 68 años proveniente de Putumayo, Colombia, quien consultó por presentar un cuadro clínico de 4 meses de evolución de dispepsia, sensación de plenitud gástrica, hiporexia, náuseas y pérdida de peso de aproximadamente $10 \mathrm{~kg}$ en 4 meses. Entre sus antecedentes refiere tabaquismo pesado hasta hace 25 años y antecedente familiar de padre con cáncer de páncreas. Al examen físico regular del estado general se encontró enflaquecido, sin adenopatías supraclaviculares, sin masas abdominales y sin ascitis. La esofagogastroduodenoscopia inicial extrainstitucional reportó engrosamiento de los pliegues fundocorporales con disminución de la distensibilidad y múltiples lesiones ulceradas superficiales. El estudio histopatológico traído de su sitio de referencia confirmó que se trata de un adenocarcinoma con patrón histológico mixto, predominantemente difuso con células en anillo de sello y $30 \%$ de tipo intestinal, pobremente diferenciado, infiltrante y ulcerado. Una tomografía de abdomen con contraste que trajo de su sitio de remisión mostró engrosamiento anormal de las paredes del estómago en la región fundocorporal de apariencia neoplásica con ulceración y adenomegalias en el ligamento gastrohepático sin compromiso hepático ni peritoneal. Se realizó un estadiaje clínico cT3N1M0.

El paciente fue llevado a cirugía con la intención de una gastrectomía con base en la evaluación preoperatoria y dentro de la cirugía se encontró una lesión gástrica con engrosamiento de la región corporal y de la curvatura menor, con un conglomerado ganglionar sobre la curvatura menor y múltiples nódulos hepáticos de aspecto metastásico, catalogándose como un estadio IV por T3N1M1. Luego de la cirugía se realizó una nueva tomografía axial computarizada (TAC) institucional y se confirmaron los hallazgos 
quirúrgicos, con múltiples lesiones focales hipodensas de $10 \mathrm{~mm}$ en los segmentos IV y VI del hígado (Figura 1A y B), con engrosamiento difuso de las paredes del estómago y conglomerado ganglionar de $31 \times 17 \mathrm{~mm}$.

Por tratarse de un estadio avanzado metastásico se inició quimioterapia con protocolo XP (cisplatino + capecitabina) y después del primer ciclo se recibió el reporte de la biopsia hepática de la institución que informó hígado comprometido por un adenocarcinoma gástrico metastásico HER2 positivo 3+. Por esta razón, se añadió trastuzumab al tratamiento instaurado, combinación que recibió por 4 ciclos más, cuando se decidió suspender el tratamiento por toxicidad caracterizada por astenia, adinamia, hiporexia, náuseas, emesis y diarrea hasta grado 3. En ese momento se realizaron nuevas tomografías de tórax y abdomen en las que se observó persistencia del engrosamiento de la cámara gástrica, las adenopatías y desaparición de las lesiones hepáticas con aparente respuesta completa de los nódulos hepáticos (Figura 1C).

Se decidió continuar la quimioterapia con capecitabina y trastuzumab, pero se suspendió el cisplatino; y después de 2 ciclos adicionales, se realizó una laparoscopia en la que no se encontró evidencia macroscópica de metástasis hepáticas ni de carcinomatosis peritoneal, por lo que en junta de cirugía se decidió el manejo quirúrgico con una intención potencialmente curativa.

Antes de la nueva intervención quirúrgica propuesta, recibió dos dosis de trastuzumab como monoterapia. Finalmente, fue llevado a cirugía el 27 de noviembre del 2017, 10 meses después del diagnóstico inicial y se realizó una gastrectomía total más vaciamiento de la segunda cadena ganglionar (D2). La patología definitiva mostró un adenocarcinoma gástrico de patrón mixto en estado de posneoadyuvancia (grado 2 de regresión) con bordes de sección libres de tumor y posi-

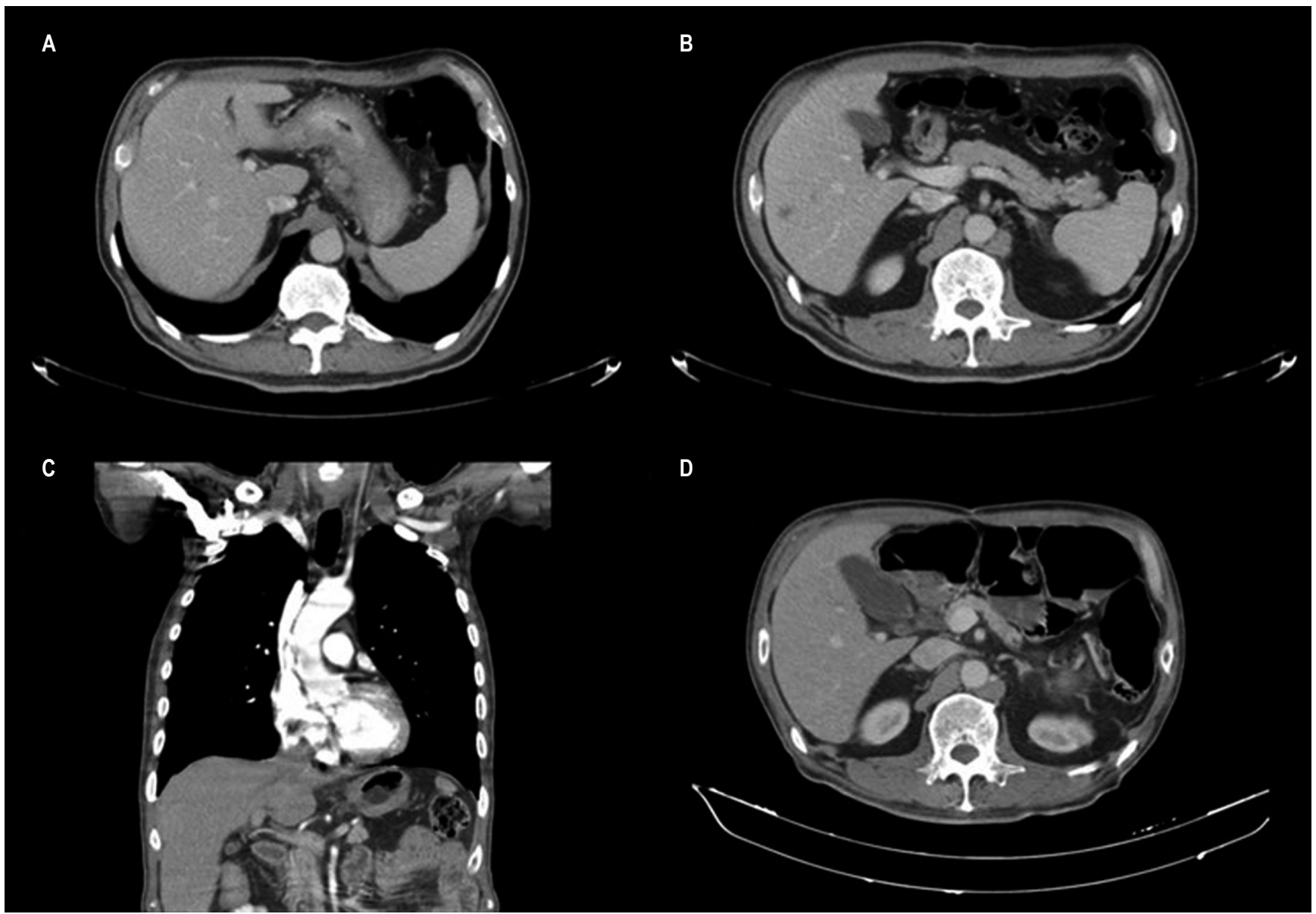

Figura 1. Estudios imagenológicos por TAC para el seguimiento de patología tumoral. A. Engrosamiento difuso de las paredes del estómago además del conglomerado ganglionar por extensión extramural de dimensiones 31 x $17 \mathrm{~mm}$. B. Lesiones hepáticas focales hipodensas en segmentos IV y VI hepáticos de hasta $10 \mathrm{~mm}$. C. Disminución de engrosamiento de cámara gástrica y adenopatías, no se observan nódulos hepáticos ni lesiones nuevas. D. Cambios posgastrectomía, hígado de tamaño, morfología, densidad y contornos normales, sin lesiones focales ni otras lesiones de extensión tumoral. 
tividad de 3/33 ganglios linfáticos para metástasis; estado HER2 positivo (3+) en el espécimen quirúrgico (Figura 2).

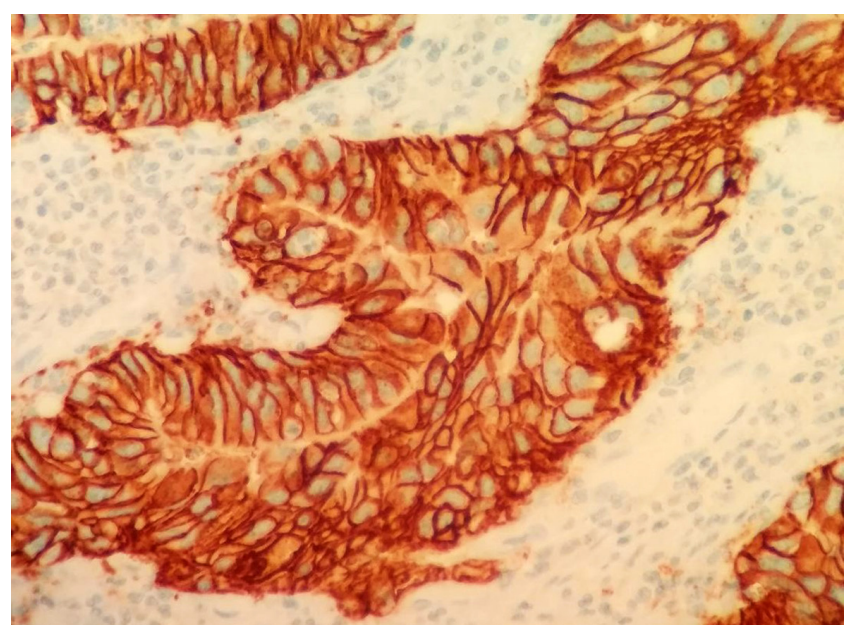

Figura 2. Adenocarcinoma gástrico HER2 positivo 3+. IHC 40x (en material de gastrectomía total).

Después de la cirugía, el paciente recibió quimioterapia adyuvante con capecitabina, oxaliplatino y trastuzumab por 4 ciclos, y luego trastuzumab en monoterapia por otros 4 ciclos más, para un total de 18 ciclos de trastuzumab perioperatorio.

5 meses después de la cirugía, una nueva TAC toracoabdominal (el 11 de abril de 2018) reportó hígado de tamaño, morfología, densidad y contornos normales, sin lesiones focales y sin otras lesiones de extensión tumoral (Figura 1D). Posteriormente, asistió a controles y se encontró asin- tomático con leve hipoacusia y cambios de pigmentación en la piel por la quimioterapia. Una tomografía por emisión de positrones (PET scan) tomada el 17 de septiembre de 2018 no mostró evidencia de lesiones tumorales (Figura 3) y una resonancia magnética nuclear del 28 de octubre de 2018 tampoco evidenció lesiones sospechosas de malignidad en la anastomosis esofagoyeyunal ni lesiones a nivel del parénquima hepático. 18 meses después del diagnóstico inicial, el paciente se encuentra asintomático y no tiene signos que indiquen recaída tumoral. El estudio, tratamiento y seguimiento anteriormente mencionados se esquematizan en la Figura 4.

\section{DISCUSIÓN}

Existen diferentes clasificaciones para el cáncer gástrico y aún son motivo de discusión. La de la American Joint Committee on Cancer (AJCC) y la de la Unión Internacional para el Control del Cáncer (UICC) son las clasificaciones utilizadas con mayor frecuencia y se basan en el estadiaje, en el que se valora la penetración de la lesión en la pared gástrica $(\mathrm{T})$, el compromiso ganglionar (N) y el metastásico (M), y clasifica al cáncer gástrico como temprano y avanzado (12). Otras se refieren a la clasificación histopatológica, como la de Lauren (adenocarcinoma de tipo intestinal, difuso y mixto), la clasificación de Nakamura, la de la Organización Mundial de la Salud (OMS), la clasificación macroscópica endoscópica de la lesión descrita para el cáncer gástrico temprano y para el cáncer gástrico avanzado (Bormann) y la clasificación genómica o molecular (2).

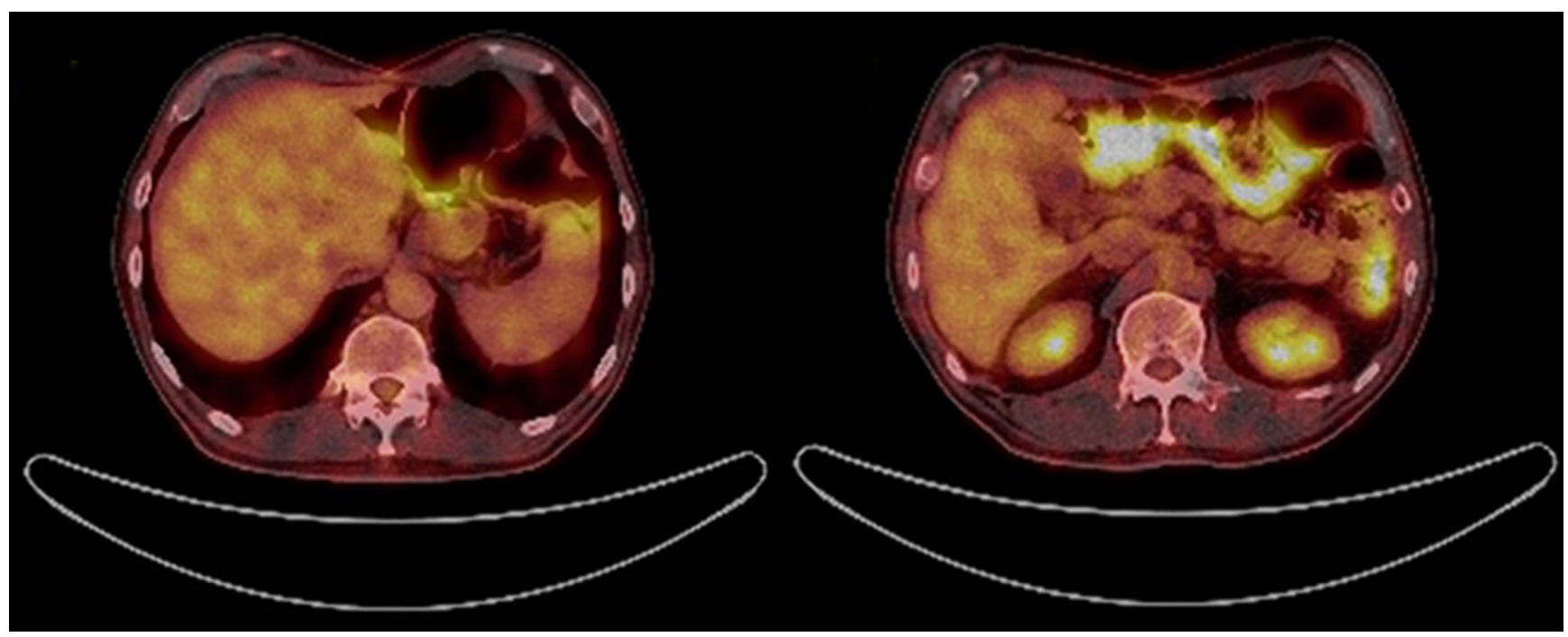

Figura 3. PET scan sin evidencia de lesiones tumorales, estado posgastrectomía. 


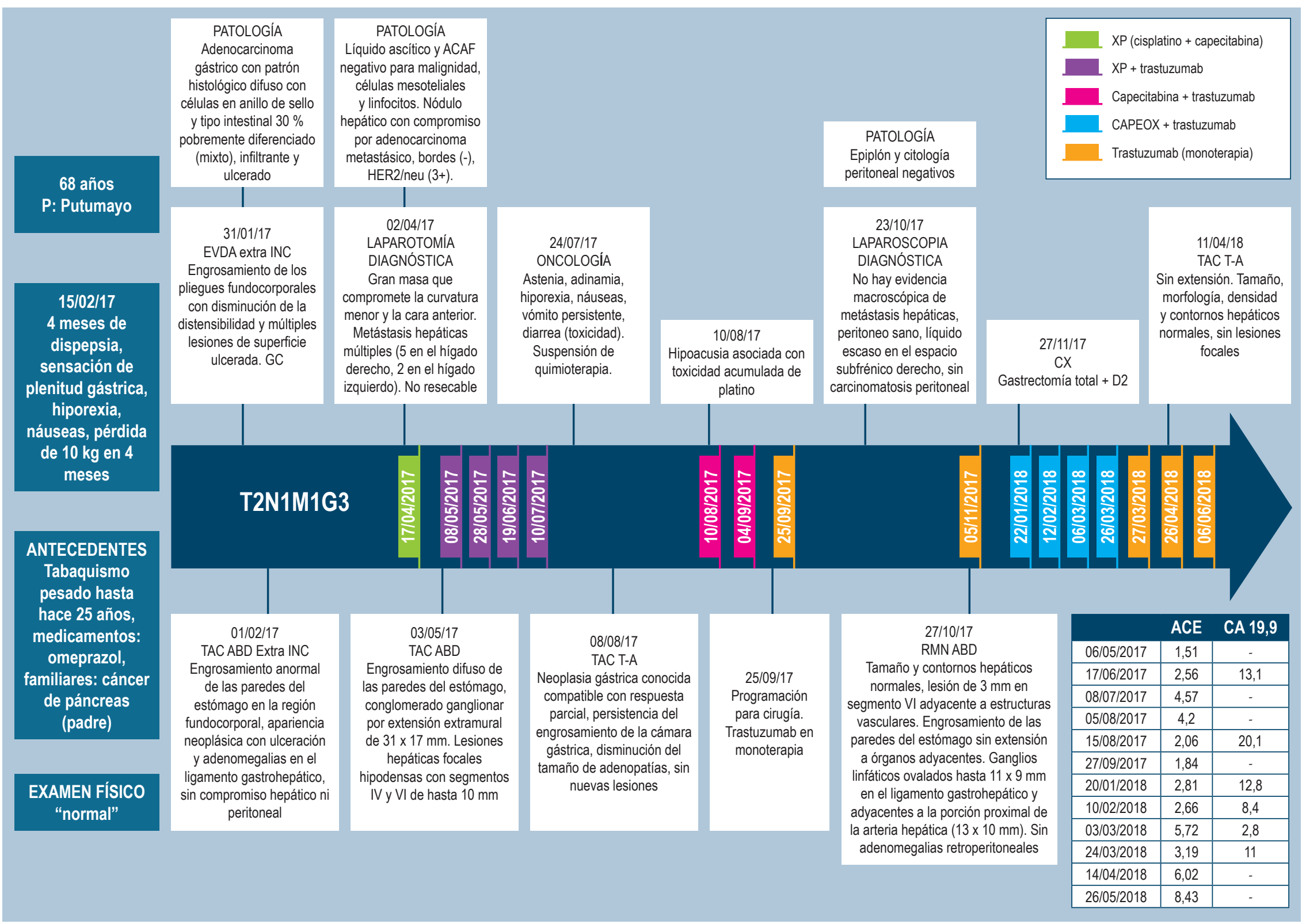

Figura 4. Línea de tiempo que esquematiza abordaje inicial, estudios, tratamiento y seguimiento del paciente presentado en este caso. ABD: abdominal; ACAF: aspiración con aguja fina; ACE: antígeno carcinoembrionario marcador tumoral; CA 19-9: marcador tumoral; CX: cirugía; EVDA: endoscopia de las vías digestivas altas; GC: cáncer gástrico; INC: Instituto Nacional de Cancerología; RMN: resonancia magnética nuclear; T-A: toracoabdominal. 
En el ámbito clínico se utiliza muy comúnmente el término de cáncer gástrico no resecable, concepto con implicaciones clínicas muy variables porque no representa la verdadera extensión de la enfermedad.

Cuando se habla de tratamiento por estadios para el cáncer gástrico se menciona como una enfermedad localizada (estadios 0, I, II y III resecable) y como una enfermedad avanzada y metastásica (estadio IV irresecable). Por esta razón, la literatura también menciona al cáncer gástrico avanzado como una entidad que incluye dos situaciones clínicas distintas: el cáncer gástrico localmente avanzado no resecable y la enfermedad metastásica (3).

El pronóstico del cáncer gástrico avanzado o metastásico es muy pobre con supervivencia a los 5 años $<4 \%$. El manejo estándar para pacientes con cáncer gástrico avanzado es la quimioterapia. Sin embargo, con la llegada de las terapias blanco es posible seleccionar el tratamiento basado en las características moleculares de la enfermedad $(2,8)$.

El cáncer gástrico fue clasificado por Pekka Lauren en 1965 con criterios histopatológicos en 2 entidades diferentes: el tipo intestinal y el tipo difuso. Antes del descubrimiento del HER2 y la introducción del trastuzumab en la práctica clínica, no existían biomarcadores predictores que permitieran dirigir el tratamiento (7).

El HER2 es un receptor de tirosina-cinasa que pertenece a la familia del EFGR codificado por el protooncogén ERBB2 en el cromosoma 17, el cual juega un papel muy importante en la diferenciación, supervivencia, apoptosis y proliferación celular (6). Sobre este receptor del factor de crecimiento epidérmico 2 , también conocido como HER2/neu, HER2, c-er-B2 y ErbB2, ha existido un interés cada vez mayor $(13,14)$.

El HER2, al tratarse de un correceptor, no tiene un ligando identificado, que sí presentan los demás miembros de la familia de receptores (Figura 5). La homodimerización genera un cambio conformacional del receptor que estimula las vías de señalización intracelular acopladas al HER2 que ocurren ya sea por la vía de la proteína cinasa activada por mitógenos (MAPK), relacionada con proliferación, migración, diferenciación o angiogénesis tumoral, o por la vía PI3K-AKT-mTOR, relacionada con supervivencia tumoral y señalización antiapoptótica (Figura 5) $(13,15)$.

El HER2 está sobreexpresado en diferentes clases de tumores que promueven la proliferación celular, progre-

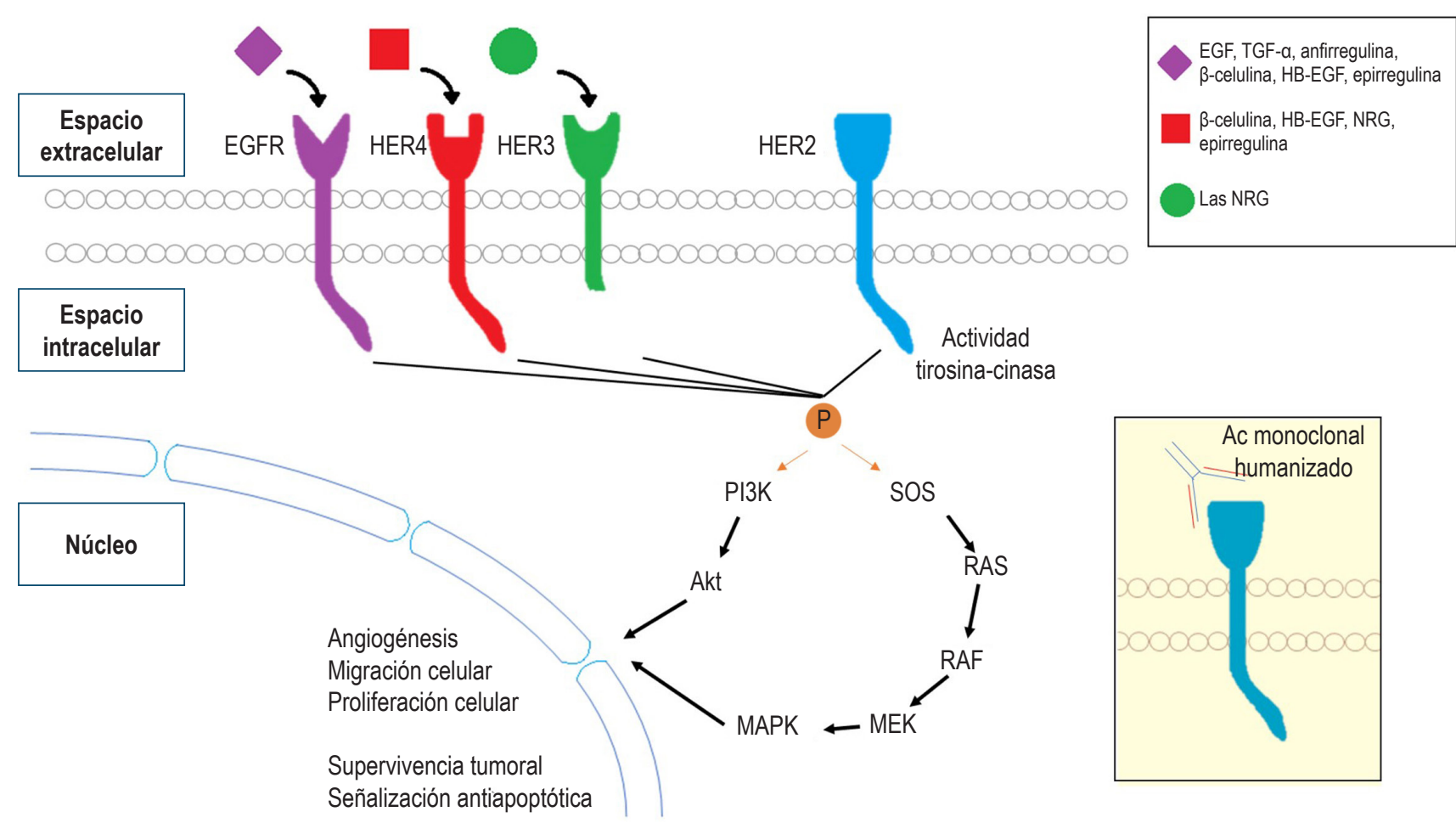

Figura 5. Interacción entre los receptores con actividad de tirosina-cinasa. Se esquematizan los diferentes receptores y en la esquina superior derecha se listan los diferentes ligandos que se relacionan con cada receptor. HER2 no cuenta con ligandos específicos, por lo que debe unirse a los demás receptores para desencadenar las vías de señalización intracelular que actuarán sobre la proliferación y supervivencia tumoral. El trastuzumab inhibe esta interacción entre HER2 y los demás miembros de la familia de receptores. AC. Akt: actividad tirosina-cinasa; HB-EGF: factor de crecimiento epidérmico ligado a heparina; NRG: neurregulina; PI3K: fosfatidilinositol-3-cinasa; TGF- $\alpha$ : factor de crecimiento transformante alfa. Elaborado por los autores. 
sión tumoral y metástasis. La alta expresión del HER2 fue detectada por primera vez en el cáncer gástrico por Fukushige y colaboradores en 1986 (16). La frecuencia de la sobreexpresión del HER2 en el cáncer gástrico varía considerablemente entre un $6 \%$ y un $30 \%$ en los diferentes estudios (6). En un intento por medir esta variabilidad, los investigadores del estudio TOGA realizaron un estudio de validación para medir con la expresión por IHC y la FISH para el estado HER en el cáncer gástrico avanzado. Las muestras de tejidos de 3807 pacientes en 24 países fueron recolectadas y analizadas utilizando IHC y FISH. El estado HER2 fue definido positivo (IHC 3+, o FISH+) con base en los patrones de tinción de membrana celular o la amplificación del gen en muestras quirúrgicas o de biopsias, respectivamente (6). La sobreexpresión del HER2 positivo estuvo asociada con el sexo masculino y con los tumores de tipo intestinal bien y moderadamente diferenciados (6).

Los criterios para definir la sobreexpresión del HER2 difieren entre el cáncer de mama y el cáncer gástrico debido a las diferencias inherentes de la biología del tumor y a las diferencias existentes en el modelo de expresión $(6,9)$. El cáncer gástrico muestra características de tinción diferentes comparadas con las del cáncer de mama, como la alta incidencia de heterogeneidad tumoral (definida como $>10 \%$ de tinción positiva de las células o solamente tinción focal de las células tumorales en grupos de más de 5 células) hasta en el $30 \%$ de los casos HER2 positivo. Otra diferencia clave con el cáncer de mama es que en los cánceres gástricos HER2 positivos las tinciones son usualmente en las glándulas de tipo intestinal y pueden mostrar una tinción incompleta, baso lateral o latero-lateral y todos estos son considerados como resultados positivos con la IHC. Por este motivo, los protocolos específicos para el examen del HER2 en el cáncer gástrico se han desarrollado y estandarizado, y es imperativo que estas recomendaciones se sigan $(2,9)$.

Dadas estas diferencias entre la expresión entre el cáncer de mama y el gástrico, se desarrolló un apropiado sistema de puntaje exclusivo para tumores gástricos. Este sistema propuesto por Hofmann y colaboradores ha sido adoptado y es específico tanto para los tumores gástricos como también para las biopsias de espécimen quirúrgico $(2,17)$.

La heterogeneidad intratumoral parece igualmente tener resultados conflictivos de la expresión del HER2 en las muestras del tumor primario y de las metástasis. En estudios previos se han encontrado diferencias significativas cuando se analiza la expresión del HER2 en secciones de tejido completo y en muestras de tejido pequeñas. Por ello es prudente extender la evaluación en más de una muestra o en todos los especímenes disponibles y, si es factible, también evaluar las metástasis. Entonces, se recomienda que los pacientes con resultados HER2 negativos en biopsias deban ser examinados en los especímenes quirúrgicos para incrementar la posibilidad de hallar tumores HER2 positivos (17). Las biopsias gástricas por endoscopia deben ser múltiples idealmente entre 6 y 8 fragmentos. Las guías alemanas recomiendan un mínimo de 8 muestras $(6,18,19)$. El estudio con IHC debe ser la prueba inicial y las pruebas moleculares como la FISH (CISH o DISH) deben ser utilizadas para reevaluar los resultados de la prueba IHC $2+$, o equívocos $(2,6,9)$.

Con base en los resultados del estudio TOGA, el trastuzumab fue aprobado para cáncer gástrico HER2 positivo, el cual es definido como IHC $3+o$ FISH positivo en los Estados Unidos y Japón. Por el contrario, en Europa, el cáncer gástrico HER2 positivo es definido como IHC $3+o$ como IHC 2+ más FISH positivo (6).

Las guías para el estado del HER2 desarrolladas por la Sociedad Japonesa de Patología recomiendan que la prueba para HER2 debe ser rutinariamente realizada en pacientes con cáncer gástrico metastásico o recurrente al momento del diagnóstico inicial $(6,9)$.

El algoritmo de la prueba desarrollada para el estado HER2 incluye primero la IHC seguida por FISH para los pacientes con IHC 2+ (Figura 6) (17).

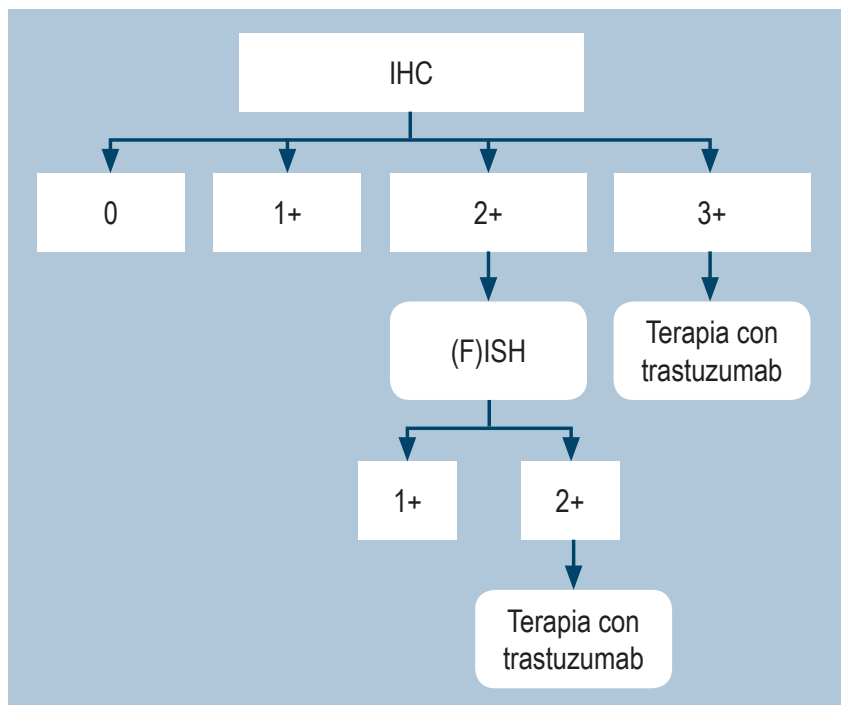

Figura 6. Algoritmo histopatológico para determinar la sobreexpresión del HER2 (18). Tomado de: Lordick F et al. J Cancer Res Clin Oncol. 2017;143(5):835-841.

El trastuzumab es un anticuerpo monoclonal humanizado que se une selectivamente al HER2, el cual está indicado para el tratamiento de tumores de mama y gástricos HER2 positivo (11). En 2010 la Agencia Europea de medicamentos (EMA) y la Food and Drug Administration (FDA) aprobaron el trastuzumab con base en el beneficio de la supervivencia global para pacientes con cáncer gástrico metastásico o cáncer de la unión gastroesofágica 
que tengan sobreexpresión del HER2 determinada por una medición exacta y validada $(9,11)$.

El estudio TOGA (20), publicado en 2010, fue un estudio prospectivo de fase III en el cual los pacientes con cáncer gástrico avanzado HER2 positivo o de la unión gastroesofágica fueron aleatorizados para recibir trastuzumab en combinación con quimioterapia o quimioterapia sola. La quimioterapia fue administrada cada 3 semanas por 6 ciclos. El trastuzumab fue administrado en dosis de 8 $\mathrm{mg} / \mathrm{kg}$ en el día 1 del primer ciclo y luego $6 \mathrm{mg} / \mathrm{kg}$ cada 3 semanas hasta la progresión de la enfermedad, toxicidad no aceptable o suspensión por consentimiento. 3803 pacientes fueron elegidos para el estudio. Las características de los pacientes en el grupo de trastuzumab más quimioterapia $(\mathrm{n}=294)$ y quimioterapia sola $(\mathrm{n}=290)$ fueron similares, incluidas la edad, sexo, regímenes de quimioterapia, sitio del tumor (gástrico y de la unión gastroesofágica). El $97 \%$ de los pacientes en ambos grupos presentaba enfermedad metastásica y poco menos de la mitad se clasificó como FISH + / IHC 3+ (45 \% frente al $43 \%)$.

El desenlace principal del trabajo era la supervivencia global, la cual fue más larga de forma significativa en los pacientes que recibieron trastuzumab más quimioterapia en comparación con solo quimioterapia, con un incremento de 2,7 meses (13,8 meses frente a 11 meses). La supervivencia libre de progresión también fue mayor en el grupo de trastuzumab más quimioterapia (6,7 meses frente a 5,5 meses). Las cifras de respuesta total en el grupo de trastuzumab más quimioterapia fue del $47 \%$, (respuesta completa $5 \%$, respuesta parcial $42 \%$ ). La duración de la respuesta también fue mayor para el grupo de trastuzumab más quimioterapia (6,9 meses frente a 4,8 meses) (20).

En el análisis de subgrupos de pacientes también se observó que la supervivencia global fue más larga en pacientes con alta expresión del HER2 determinada por IHC y FISH (IHC 3+ o IHC 2+ más FISH positivo) que en pacientes con expresión baja del HER2 (IHC 0 o 1 + más FISH positivo) $(6,20)$. De acuerdo con estos resultados, la terapia de trastuzumab es recomendada para pacientes con IHC 3+ o IHC 2+ más FISH positivo (alta expresión de HER2) (6).

Los resultados de este estudio muestran que el trastuzumab en combinación con la quimioterapia mejora significativamente la supervivencia global en pacientes con cáncer gástrico avanzado y de la unión gastroesofágica con HER2 positivo, comparado con la quimioterapia sola, y esta mejoría fue particularmente significativa en pacientes con alta expresión del HER2. Llama la atención que el trastuzumab no incrementó la incidencia de eventos adversos asociados con la quimioterapia y que la frecuencia de eventos cardíacos fue baja (20).
Varios casos reportados han mostrado resultados favorables del trastuzumab como parte de la quimioterapia perioperatoria para el cáncer gástrico $(16,21)$. Algunos pacientes han mostrado respuesta patológica completa.

En nuestro caso reportado, el paciente fue diagnosticado con sobreexpresión del HER2 positivo en la biopsia tomada de la lesión metastásica hepática cuando se realizó la laparotomía. El reporte de la patología gástrica con el cual fue remitido no tenía reporte del estado HER2. Como se trataba de un estado avanzado por lesión gástrica con metástasis hepáticas, el paciente recibió quimioterapia estándar con capecitabina más cisplatino y, una vez conocido su estado de HER2, se adicionó el trastuzumab. Este medicamento (trastuzumab) combinado con la quimioterapia (fluoropirimidina y platino) prolonga la supervivencia global en pacientes con cáncer gástrico HER2 positivos no resecables $(2,20)$.

Dada la respuesta favorable al tratamiento instaurado por la desaparición de las lesiones hepáticas corroborado con imágenes y por una laparoscopia, al paciente se le realizó una gastrectomía total con linfadenectomía D2 con intención curativa. El resultado de la sobreexpresión del HER2 fue positivo en el espécimen quirúrgico. Después de la cirugía, recibió también trastuzumab como monoterapia y logró tener control de su enfermedad por 18 meses desde el diagnóstico inicial, con un PET scan negativo.

Lo anterior permite entender la importancia de la determinación del estado HER2 en los pacientes con cáncer gástrico avanzado y llama la atención que la introducción del trastuzumab permite el establecimiento de una nueva entidad de cáncer gástrico con HER2 positivo, de forma similar al cáncer de mama con HER2 positivo $(4,5)$. Los pacientes con cáncer gástrico avanzado con sobreexpresión del HER2 positivo se benefician del tratamiento con la terapia anti-HER2, trastuzumab (2).

En Colombia existe una alta carga de morbimortalidad por el cáncer gástrico y ya se completó más de una década del desarrollo de terapias dirigidas. Sin embargo, la literatura nacional que aborde este campo de acción específico es escasa. Entre ellos se encuentra un estudio realizado en Bucaramanga y publicado en 2013, en el cual se reportó que la frecuencia de expresión del HER 2 en pacientes con adenocarcinoma gástrico es del 11,2\% (22), y es superior en comparación con otros países latinoamericanos como Brasil, Perú y México, con un 10,5\%, 9 \% y 6,5\%, respectivamente (23).

En conclusión, el trastuzumab más la quimioterapia (FP) es el tratamiento estándar para pacientes con cáncer gástrico avanzado metastásico o de la unión gastroesofágica con HER2 positivo. Todos estos cánceres deben ser examinados para revisar el estado HER2 en los bloques de para- 
fina o en muestras de biopsias del tumor primario o de las metástasis al momento del diagnóstico inicial. Las biopsias gástricas por endoscopia deben ser múltiples. El estudio con IHC debe ser la prueba inicial. Los tumores equívocos o incompletos con IHC 2+ deben ser examinados con FISH para su confirmación. El examen del estado HER2 y su puntaje deben estar con adherencia a las recomendaciones específicas para el cáncer gástrico y deben ser realizadas en un laboratorio con experiencia $(2,9)$. Las perspectivas son la evaluación del trastuzumab en el entorno perioperatorio y adyuvante de los pacientes con cáncer gástrico avanzado con HER2 positivo.

\section{REFERENCIAS}

1. Bray F, Ferlay J, Soerjomataram I, Siegel R, Torre L, Jemal A. Global cancer statistics 2018: GLOBOCAN estimates of incidence and mortality worldwide for 36 cancers in 185 countries. Ca Cancer J Clin. 2018;68(6):394-424. https://doi.org/10.3322/caac.21492

2. Smyth EC, Nilsson M, Grabsch HI, van Grieken NC, Lordick F. Gastric cancer. Lancet. 2020;396(10251):635-648. https://doi.org/10.1016/S0140-6736(20)31288-5

3. Salati M, Di Emidio K, Tarantino V, Cascinu S. Secondline treatments: moving towards an opportunity to improve survival in advanced gastric cancer? ESMO Open. 2017;2(3):e000206.

https://doi.org/10.1136/esmoopen-2017-000206

4. Oliveros R, Facundo H, Bonilla CA, Sánchez Pedraza

$\mathrm{R}$. Terapia de conversión en Cáncer Gástrico estadio IV. A propósito de un caso. Rev Colomb Gastroenterol. 2018;33(1):66-67. https://doi.org/10.22516/25007440.233

5. Wang H B, Liao X F, Zhang J. Clinicopathological factors associated with HER2 positive gastric Cancer. A metaanalysis. Medicine 2017;96(44):1-5. https://doi.org/10.1097/MD.0000000000008437

6. Boku N. HER2-positive gastric cancer. Gastric Cancer. 2014;17(1):1-12. https://doi.org/10.1007/s10120-013-0252-z

7. Battaglin F, Naseem M, Puccini A, Lenz HJ. Molecular biomarkers in gastro-esophageal cancer: recent developments, current trends and future directions. Cancer Cell Int. $2018 ; 18: 99$. https://doi.org/10.1186/s12935-018-0594-z

8. Okita A, Imai H, Takahashi M, Takahashi H, Umegaki S, Kawamura Y, Hiraide S, Ouchi K, Sato Y, Okada Y, Komine K, Saijo K, Takahashi S, Takahashi M, Shirota H, Ohori H, Gamoh M, Ishioka C. Efficacy and Safety of Trastuzumab in Combination with S-1 and Cisplatin Therapy for Japanese Patients with HER2-Positive Advanced Gastric Cancer: Retrospective Analysis. Tohoku J Exp Med. 2018;245(2):123-129. https://doi.org/10.1620/tjem.245.123

9. Rüschoff J, Hanna W, Bilous M, Hofmann M, Osamura RY, Penault-Llorca F, van de Vijver M, Viale G. HER2 testing in gastric cancer: a practical approach. Mod Pathol. 2012;25(5):637-50.

https://doi.org/10.1038/modpathol.2011.198
10. Charalampakis N, Economopoulou P, Kotsantis I, Tolia M, Schizas D, Liakakos T, Elimova E, Ajani JA, Psyrri A. Medical management of gastric cancer: a 2017 update. Cancer Med. 2018;7(1):123-133. https://doi.org/10.1002/cam4.1274

11. Van Cutsem E, Bang YJ, Feng-Yi F, Xu JM, Lee KW, Jiao SC, Chong JL, López-Sanchez RI, Price T, Gladkov O, Stoss O, Hill J, Ng V, Lehle M, Thomas M, Kiermaier A, Rüschoff J. HER2 screening data from ToGA: targeting HER2 in gastric and gastroesophageal junction cancer. Gastric Cancer. 2015; 18(3):476-84. https://doi.org/10.1007/s10120-014-0402-y

12. Fugazzola P, Ansaloni L, Sartelli M, Catena F, Cicuttin E, Leandro G, De’ Angelis GL, Gaiani F, Di Mario F, Tomasoni M, Coccolini F. Advanced gastric cancer: the value of surgery. Acta Biomed. 2018;89(8-S):110-116. https://doi.org/10.23750/abm.v89i8-S.7897

13. Ciardello F, Tortora G. EGFR antagonists in cancer treatment. N Engl J Med. 2008;358(11):1160-1174. https://doi.org/10.1056/NEJMra0707704

14. Schechter AL, Stern DF, Vaidyanathan L, Decker SJ, Drebin JA, Greene MI, Weinberg RA. The neu oncogene: an erbB-related gene encoding a 185,000-Mr tumour antigen. Nature. 1984;312(5994):513-6. https://doi.org/10.1038/312513a0

15. Hudis C. Trastuzumab - Mechanism of action and use in clinical practice. N Engl J Med. 2007;357(1):39-51. https://doi.org/10.1056/NEJMra043186

16. Dong CX, Fu JF, Ye XY, Li XF, Zhong X, Yuan Y. Surgical resection of advanced gastric cancer following trastuzumab/oxaliplatin/capecitabine combination therapy. World J Gastroenterol. 2014 Sep 14;20(34):12355-8. https://doi.org/10.3748/wjg.v20.i34.12355

17. Abrahao-Machado LF, Scapulatempo-Neto C. HER2 testing in gastric cancer: An update. World J Gastroenterol 2016;22(19):4619-4625. https://doi.org/10.3748/wjg.v22.i19.4619

18. Lordick F, Al-Batran SE, Dietel M, Gaiser T, Hofheinz RD, Kirchner T, Kreipe HH, Lorenzen S, Möhler M, Quaas A, Röcken C, Rüschoff J, Tannapfel A, Thuss-Patience $\mathrm{P}$, Baretton G. HER2 testing in gastric cancer: results of a German expert meeting. J Cancer Res Clin Oncol. 2017; 143(5):835-841. https://doi.org/10.1007/s00432-017-2374-x 
19. Bouché O, Penault-Llorca F. HER2 et cancer de l'estomac: une nouvelle cible thérapeutique pour le trastuzumab. Bull Cancer. 2010;97(12):1429-40.

https://doi.org/10.1684/bdc.2010.1224

20. Bang YJ, Van Cutsem E, Feyereislova A, Chung HC, Shen L, Sawaki A, Lordick F, Ohtsu A, Omuro Y, Satoh T, Aprile G, Kulikov E, Hill J, Lehle M, Rüschoff J, Kang YK; ToGA Trial Investigators. Trastuzumab in combination with chemotherapy versus chemotherapy alone for treatment of HER2-positive advanced gastric or gastro-oesophageal junction cancer (ToGA): a phase 3, open-label, randomised controlled trial. Lancet. 2010;376(9742):687-97. https://doi.org/10.1016/S0140-6736(10)61121-X

21. Wang J, Saukel GW, Garberoglio CA, Srikureja W, Hsueh CT. Pathological complete response after neoadjuvant che- motherapy with trastuzumab-containing regimen in gastric cancer: a case report. J Hematol Oncol. 2010;3:31. https://doi.org/10.1186/1756-8722-3-31

22. García C, Uribe C, Niño P, Salazar D, Vásquez L. Expresión de Her2/neu en carcinoma gástrico en el área metropolitana de Bucaramanga en el periodo 2006 a 2009. Méd UIS. 2013;26(2):21-28.

23. Alvarado I, Gil S, Ruelas A, Villaverde D, Montes J, Medrano R. Evaluación por inmunohistoquímica de la expresión del HER2 en cáncer gástrico. Estudio clínico-patológico de 93 casos. Cirugía y Cirujanos. 2017;85(6):504-509.

https://doi.org/10.1016/j.circir.2016.11.016 